X. ZHANG, X. ZHAO AND S. WANG

KODAI MATH. J.

38 (2015), 451-469

\title{
SOVEREIGN AND RIBBON WEAK HOPF ALGEBRAS
}

\author{
Xiaohui Zhang, Xiaofan Zhao and Shuanhong Wang
}

\begin{abstract}
In this paper, we study the Deligne's sovereign structure theorem on the finite dimensional weak Hopf algebras, and give a necessary and sufficient condition for a finite dimensional (co)quasitriangular weak Hopf algebra $H$ with bijective antipode to admit a (co)ribbon structure. As an application we discuss the ribbon structures over the Drinfeld doubles of some weak Hopf algebras to verify our theory.
\end{abstract}

\section{Introduction}

In 1996, Böhm and Szlachányi ([3]) introduced and studied weak Hopf algebras (or quantum groupoids) as a generalization of ordinary Hopf algebras and groupoid algebras. The axioms are the same as the ones for a Hopf algebra, except that the coproduct of the unit, the product of counit and the antipode condition are replaced by the weaker properties. General theory for the weak Hopf algebras was subsequently developed in [3], [14], [15], [16], etc..

A ribbon structure (see [10], and also see [18]) in a rigid braided category $\mathscr{C}$ is a self-dual twist (or a self-dual balanced structure), which is a natural isomorphism from the identity functor to itself and compatible with the duality and the braiding. A sovereign structure (or a pivotal structure) in an autonomous category (see [1], and also see [8]) is a monoidal natural isomorphism from the left duality functor to the right duality functor. In 1992, Deligne ([20], Proposition 2.11) showed that there is a twist in a rigid braided category $\mathscr{C}$ if and only if $\mathscr{C}$ admits a sovereign structure. Thus a ribbon structure in $\mathscr{C}$ must be a sovereign structure satisfying some axioms. From the reconstruction theoretical point of view, a ribbon (resp. sovereign) category is equivalent to the category of (co)modules over a (co)ribbon (resp. sovereign) Hopf algebras (or its generalizations) (see [1], [4], [17]). This leads us to a natural question: how to represent the Deligne's Theorem in the Hopf algebra (or its generalizations) language?

2010 Mathematics Subject Classification. 16T05; 16W30.

Key words and phrases. ribbon weak Hopf algebra; sovereign element; cosovereign weak Hopf algebra; Drinfeld double.

Received April 30, 2014; revised November 28, 2014. 
In 1993, Kauffman and Radford ([11], Theorem 1) gave a bijective map between the ribbon elements and the sovereign elements (notice that they did not use the word "sovereign") satisfying suitable axioms related with the Drinfeld element in a quasitriangular Hopf algebra. That provides us a Hopf algebra view of Deligne's Theorem (also see Theorem 1.3, [6]). In 2001, Bichon ([1]) introduced the (co)sovereign Hopf algebras and described the relation between the cosovereign Hopf algebras and coquasitriangular Hopf algebras. In 2007, Bruguières and Virelizier provided the Hopf monad version of Deligne's Theorem (see [4], Theorem 8.14). A natural idea is to discuss how this theorem appears in a weak Hopf algebra version. This is the motivation of our paper.

In this paper, we first review some basic definitions. In Section 2, we discuss the relation between the ribbon elements and sovereign elements in a weak Hopf algebra, and give the definition of a (co)sovereign weak Hopf algebra. As the main result of this section, we mainly find a necessary and sufficient condition based on Deligne's Theorem for a (co)quasitriangular weak Hopf algebra to be (co)ribbon. Finally, as an application, we consider the Drinfeld doubles for the 2-dimensional, 3-dimensional and Sweedler's 5-dimensional weak Hopf algebras (note that these examples are in fact face algebras introduced by Hayashi in [9]), and discuss whether they admit the ribbon structures or sovereign structures.

\section{Preliminaries}

Throughout this article, we always work over a fixed field $k$ satisfying char $k \neq 2$. All algebras, linear spaces etc. will be taken over $k$. We use Sweedler's natation for coproduct on a coalgebra $H: \Delta(h)=h_{1} \otimes h_{2}$, for all $h \in H$, where summation is understood.

In this section we recall some basic definitions and results related to our paper.

\subsection{Sovereign categories and ribbon categories}

Let $(\mathscr{C}, \otimes, I, a, l, r)$ and $\left(\mathscr{C}^{\prime}, \otimes^{\prime}, I^{\prime}, a^{\prime}, l^{\prime}, r^{\prime}\right)$ be two monoidal categories. A natural transformation $\alpha: F \Rightarrow G: \mathscr{C} \rightarrow \mathscr{C}^{\prime}$ is called monoidal if $\alpha$ satisfies

$$
\alpha_{X \otimes Y} \circ F_{2}(X, Y)=G_{2}(X, Y) \circ\left(\alpha_{X} \otimes \alpha_{Y}\right), \quad \text { and } \quad G_{0}=\alpha_{I} F_{0},
$$

or equivalently ([7], Definition 1.5.1), if $\alpha$ satisfies

$\alpha_{X \otimes Y} \circ F_{2}(X, Y)=G_{2}(X, Y) \circ\left(\alpha_{X} \otimes \alpha_{Y}\right), \quad$ and $\alpha_{I}$ is an isomorphism.

DEFINITION 1.1. A sovereign structure on an autonomous category $\mathscr{C}$ is a monoidal natural isomorphism $\gamma:()^{*} \rightarrow^{*}()$, where ()$^{*}$ means the left dual functor and ${ }^{*}()$ means the right dual functor. A sovereign category is an autonomous category with a sovereign structure.

Note that there is another definition of a sovereign structure in [8] and [20] which used the monoidal natural isomorphism from ${ }^{*}()$ to ()$^{*}$. 
Recall from [1] that in the earlier definition by Freyd and Yetter (see [8], [20]) there is a redundant axiom, which was proved by Maltsiniotis (see [13], Proposition 3.2.3).

Recall from [[19], Remark 4.12] that one can equivalently define a sovereign category as an autonomous category equipped with a monoidal natural transformation $i: i d \rightarrow()^{* *}$ (in [8] and [19] it is called a pivotal structure).

Let $(\mathscr{C}, \otimes, I, a, l, r, c)$ be a braided monoidal category. Recall from [10] (or [19]) that a twist (or a balanced structure) on $\mathscr{C}$ is a family $\theta_{V}: V \rightarrow V$ of natural isomorphisms indexed by the objects $V$ of $\mathscr{C}$ satisfying

$$
\theta_{V \otimes W}=c_{W, V} c_{V, W}\left(\theta_{V} \otimes \theta_{W}\right) .
$$

A twist $\theta$ on an autonomous category $\mathscr{C}$ is self-dual if $\theta_{V^{*}}=\left(\theta_{V}\right)^{*}$ (or equivalently, $\left.\theta^{*} V={ }^{*}\left(\theta_{V}\right)\right)$.

A ribbon category is a braided autonomous category endowed with a selfdual twist.

Deligne's Theorem ([20], Proposition 2.11, or [19], Lemma 4.20). Let $\mathscr{C}$ be a braided autonomous category. Then giving a twist $\theta_{V}: V \rightarrow V$ on $\mathscr{C}$ (making $\mathscr{C}$ into a balanced category) is equivalent to giving a sovereign structure $i_{V}: V^{*} \rightarrow{ }^{*} V$ (making $\mathscr{C}$ into a sovereign category).

\section{2. (Co)Ribbon weak Hopf algebras}

Recall from [3] that a weak Hopf algebra $(H, m, \eta, \Delta, \varepsilon, S)$ is both an associative algebra and a coassociative coalgebra with an antipode $S: H \rightarrow H$ satisfying the following conditions: for all $x, y, z \in H$,

$$
\left\{\begin{array}{l}
(1) \Delta(x y)=\Delta(x) \Delta(y) \\
(2) \Delta^{2}(1)=(\Delta(1) \otimes 1)(1 \otimes \Delta(1))=(1 \otimes \Delta(1))(\Delta(1) \otimes 1) \\
(3) \varepsilon(x y z)=\varepsilon\left(x y_{1}\right) \varepsilon\left(y_{2} z\right)=\varepsilon\left(x y_{2}\right) \varepsilon\left(y_{1} z\right) ; \\
(4) x_{1} S\left(x_{2}\right)=\varepsilon\left(1_{1} x\right) 1_{2}, \quad S\left(x_{1}\right) x_{2}=1_{1} \varepsilon\left(x 1_{2}\right), \quad S\left(x_{1}\right) x_{2} S\left(x_{3}\right)=S(x) .
\end{array}\right.
$$

For a weak bialgebra $H$, define the maps $\varepsilon_{t}, \varepsilon_{s}: H \rightarrow H$ by the formulas

$$
\varepsilon_{t}(x)=\varepsilon\left(1_{1} x\right) 1_{2} ; \quad \varepsilon_{s}(x)=1_{1} \varepsilon\left(x 1_{2}\right) .
$$

Denote the image $\varepsilon_{t}(H)$ by $H_{t}$ and the image $\varepsilon_{s}(H)$ by $H_{s}$, where $H_{t}$ and $H_{s}$ are called the target algebra and source algebra of $H$.

Recall from [14] that an element $g \in H$ is said to be group-like if it is invertible and satisfies

$$
\Delta(g)=\Delta(1)(g \otimes g)=(g \otimes g) \Delta(1) .
$$

Group-like elements of $H$ form a group denoted by $G(H)$. Note that for any $g \in G(H)$, we have $\varepsilon_{t}(g)=\varepsilon_{s}(g)=1$ and the element $S(g)=g^{-1}$ is also grouplike. 
Suppose that $H$ is a finite dimensional weak Hopf algebra. Then the linear dual $H^{*}$ is also a finite dimensional weak Hopf algebra with structure maps $\hat{m}$, $\hat{\eta}, \hat{\varepsilon}, \hat{\Delta}, \hat{S}$ (see [3]).

A quasitriangular weak Hopf algebra $([16])$ is a pair $(H, R)$ where $H$ is a weak Hopf algebra and $R=\sum R^{(1)} \otimes R^{(2)} \in \Delta^{o p}(1)(H \otimes H) \Delta(1)$ such that

(1) there exists $\bar{R} \in \Delta(1)(H \otimes H) \Delta^{o p}(1)$ with

$$
R \bar{R}=\Delta^{o p}(1), \quad \bar{R} R=\Delta(1) ;
$$

(2) for all $h \in H$, we have

$$
\left\{\begin{array}{l}
\Delta^{o p}(h) R=R \Delta(h), \\
(i d \otimes \Delta) R=R_{13} R_{12}, \\
(\Delta \otimes i d) R=R_{13} R_{23},
\end{array}\right.
$$

where $R_{12}=R \otimes 1, R_{23}=1 \otimes R$, etc.

Let $(H, R)$ be a quasitriangular weak Hopf algebra. If there is an invertible central element $v \in H$, such that

$$
\Delta(v)=R_{21} R(v \otimes v) \text { and } S(v)=v,
$$

then $v$ is called a ribbon element and $H$ is called a ribbon weak Hopf algebra.

A coquasitriangular weak Hopf algebra $([5])(H, \sigma)$ consists of a weak Hopf algebra $H$ and a map $\sigma: H \otimes H \rightarrow k$ satisfying the following conditions:

(1) there exists a map $\bar{\sigma}: H \otimes H \rightarrow k$ (called a weak inverse of $\sigma$ ) such that

$$
\varepsilon\left(a_{1} b_{1}\right) \bar{\sigma}\left(a_{2} \otimes b_{2}\right) \varepsilon\left(b_{3} a_{3}\right)=\bar{\sigma}(a \otimes b),
$$

and

$$
\sigma\left(a_{1} \otimes b_{1}\right) \bar{\sigma}\left(a_{2} \otimes b_{2}\right)=\varepsilon(b a), \quad \bar{\sigma}\left(a_{1} \otimes b_{1}\right) \sigma\left(a_{2} \otimes b_{2}\right)=\varepsilon(a b) ;
$$

(2) for all $a, b, c \in H$, we have

$$
\left\{\begin{array}{l}
\sigma\left(a_{1} \otimes b_{1}\right) a_{2} b_{2}=b_{1} a_{1} \sigma\left(a_{2} \otimes b_{2}\right), \\
\sigma(a \otimes b c)=\sigma\left(a_{1} \otimes c\right) \sigma\left(a_{2} \otimes b\right), \\
\sigma(a b \otimes c)=\sigma\left(a \otimes c_{1}\right) \sigma\left(b \otimes c_{2}\right), \\
\varepsilon\left(b_{1} a_{1}\right) \sigma\left(a_{2} \otimes b_{2}\right) \varepsilon\left(a_{3} b_{3}\right)=\sigma(a \otimes b) .
\end{array}\right.
$$

Note that the above definition is opposite to Pfeiffer's one (see [17]). Actually, they are weak inverse with each other.

Let $H$ be a weak Hopf algebra. A linear form $f: H \rightarrow k$ is called we have

1. convolution invertible if there exists $f^{-1}: H \rightarrow k$ such that for any $x \in H$

$$
f\left(x_{1}\right) f^{-1}\left(x_{2}\right)=f^{-1}\left(x_{1}\right) f\left(x_{2}\right)=\varepsilon(x) ;
$$

2. dual central if $f\left(x_{1}\right) x_{2}=x_{1} f\left(x_{2}\right)$ for any $x \in H$;

3. dual group-like if it is convolution invertible and satisfies for any $x, y \in H$

$$
f(x y)=\varepsilon\left(x_{1} y_{1}\right) f\left(x_{2}\right) f\left(y_{2}\right)=f\left(x_{1}\right) f\left(y_{1}\right) \varepsilon\left(x_{2} y_{2}\right) .
$$


Let $(H, \sigma)$ be a coquasitriangular weak Hopf algebra. If there is a convolution invertible and dual central linear form $\tau: H \rightarrow k$ satisfying the following conditions

$$
\tau(x y)=\sigma\left(y_{1} \otimes x_{1}\right) \sigma\left(x_{2} \otimes y_{2}\right) \tau\left(x_{3}\right) \tau\left(y_{3}\right), \quad \text { and } \quad \tau(S(x))=\tau(x),
$$

for any $x, y \in H$, then $\tau$ is called a ribbon form of $H$, and $H$ is called a coribbon weak Hopf algebra.

\subsection{The representations of weak Hopf algebras}

Let $\operatorname{Rep}(H)$ be the category of representations of a weak Hopf algebra $H$, whose objects are finite dimensional left $H$-modules and whose morphisms are $H$-linear homomorphisms.

For any $V, W \in \operatorname{Rep}(H)$, define their tensor product by

$$
V \otimes_{t} W=\Delta\left(1_{H}\right)\left(V \otimes_{k} W\right),
$$

and the module structure is given by

$$
h \cdot(v \otimes w)=h_{1} \cdot v \otimes h_{2} \cdot w, \quad \text { for any } h \in H, v \otimes w \in V \otimes_{t} W,
$$

then recall from [15] that $\operatorname{Rep}(H)$ is a rigid category through the following statements:

- $H_{t}$ is the unit object of $\operatorname{Rep}(H)$, where the action of $H$ on $H_{t}$ is given by

$$
h \cdot x=\varepsilon_{t}(h x), \quad h \in H, x \in H_{t},
$$

and the unit constraints are given by

$$
\begin{gathered}
l_{V}: H_{t} \otimes V \rightarrow V, \quad x \otimes v \mapsto x \cdot v, \quad l_{V}^{-1}: V \rightarrow H_{t} \otimes V, \quad v \mapsto S\left(1_{1}\right) \otimes 1_{2} \cdot v, \\
r_{V}: V \otimes H_{t} \rightarrow V, \quad v \otimes x \mapsto S^{-1}(x) \cdot v, \quad r_{V}^{-1}: V \rightarrow V \otimes H_{t}, \quad v \mapsto 1_{1} \cdot v \otimes 1_{2} ;
\end{gathered}
$$

- for any $V \in \operatorname{Rep}(H)$, set $V^{*}=\operatorname{hom}_{k}(V, k)$, with the action of $H$ on $V^{*}$ given by

$$
(h \cdot f)(v)=f(S(h) \cdot v), \quad h \in H, f \in V^{*}, v \in V,
$$

and $V^{*}$ is a left dual of $V$ via

$$
\operatorname{coev}_{V}: H_{t} \rightarrow V \otimes V^{*}, \quad x \mapsto x \cdot\left(\sum q_{\alpha} \cdot e_{i} \otimes e^{i}\right),
$$

where $e_{i}$ and $e^{i}$ are bases of $V$ and $V^{*}$ respectively, dual to each other, and

$$
e v_{V}: V^{*} \otimes V \rightarrow H_{t}, \quad f \otimes v \mapsto f\left(1_{1} \cdot v\right) 1_{2} ;
$$

- for any $V \in \operatorname{Rep}(H)$, set ${ }^{*} V=\operatorname{hom}_{k}(V, k)$, with the action of $H$ on ${ }^{*} V$ given by

$$
(h \cdot f)(v)=f\left(S^{-1}(h) \cdot v\right), \quad h \in H, f \in{ }^{*} V, v \in V,
$$


and ${ }^{*} V$ is a right dual of $V$ via

$$
\widetilde{\operatorname{coev}}_{V}(x)=x \cdot\left(\sum e^{i} \otimes e_{i}\right), \quad \widetilde{e v}_{V}(v \otimes f)=f\left(S^{-1}\left(1_{1}\right) \cdot v\right) 1_{2} .
$$

Recall from [15] that a weak Hopf algebra $H$ is a quasitriangular weak Hopf algebra if and only if $\operatorname{Rep}(H)$ is a braided category with the braiding

$$
c_{V, W}: V \otimes W \rightarrow W \otimes V, \quad v \otimes w \mapsto \sum R^{(2)} \cdot w \otimes R^{(1)} \cdot v,
$$

and a weak Hopf algebra $H$ is a ribbon weak Hopf algebra if and only if $\operatorname{Rep}(H)$ is a ribbon category with the twist

$$
\theta_{V}: V \rightarrow V, \quad v \mapsto v \cdot v
$$

\section{Sovereign weak Hopf algebras and ribbon weak Hopf algebras}

Assume that $H$ is a finite dimensional weak Hopf algebra with the bijective antipode $S$. In this section, we will introduce the notion of a (co)sovereign weak Hopf algebra, and consider the (co)representation category over it and give a necessary and sufficient condition for a finite dimensional (co)quasitriangular weak Hopf algebra to admit a (co)ribbon structure through the (co)sovereign structure. This work generalizes [[11], Theorem 1] and [[1], Proposition $2.9 \&$ 2.10].

Let ()$^{*}$ be the left dual functor, ${ }^{*}()$ be the right dual functor over $\operatorname{Rep}(H)$, $F$ be the underlying functor from $\operatorname{Rep}(H)$ to $V e c_{k}$. Let $\Omega$ denote the collection of all natural transformations from $F \circ()^{*}$ to $F \circ{ }^{*}()$.

Lemma 2.1. There is a bijective map between $\Omega$ and $H$.

Proof. Define a map

$$
P: \Omega \rightarrow H, \quad i \mapsto P(i)=\gamma:=i_{H}\left(a^{i}\right)\left(1_{H}\right) a_{i},
$$

where $a_{i}$ and $a^{i}$ are bases of $H$ and $H^{*}$, respectively, dual to each other, and define

$$
Q: H \rightarrow \Omega, \quad \gamma \mapsto Q(\gamma)=i,
$$

where $\gamma \in H$ and $i$ satisfies

$$
i_{M}: M^{*} \rightarrow{ }^{*} M, \quad f \mapsto f^{\prime}, \quad \text { where } f^{\prime}(m)=f(\gamma \cdot m),
$$

for any $m \in M \in \operatorname{Rep}(H)$. It is easy to check that $i$ is a natural transformation.

Obviously $P$ and $Q$ are inverse with each other. other.

From now on, assume that $i \in \Omega$ and $\gamma \in H$ are in correspondence with each 
LeMma 2.2. $i$ is H-linear if and only if $\gamma$ satisfies

$$
S^{2}(h)=\gamma h \gamma^{-1}, \quad \text { for all } h \in H .
$$

Proof. $\Rightarrow$ : Since $i_{H}$ is $H$-linear, for any $h, x \in H, f \in H^{*}$, we have

$$
i_{H}(h \cdot f)(x)=h \cdot\left(i_{H}(f)(x)\right),
$$

which implies

$$
f\left(S^{-1}(h) \gamma x\right)=f(\gamma S(h) x) .
$$

Thus $S(h) \gamma x=\gamma S^{-1}(h) x$. Take $x=1_{H}$, we get $S^{2}(h)=\gamma h \gamma^{-1}$.

$\Leftarrow$ : Straightforward.

LEMMA 2.3. $i$ is a natural isomorphism in $\Omega$ if and only if $\gamma \in H$ is invertible.

Proof. $\Rightarrow$ : Assume that $i^{-1}:{ }^{*}() \rightarrow()^{*}$ is the inverse of $i$, then the inverse element of $\gamma$ is $\gamma^{\prime}=i_{H}^{-1}\left(a^{i}\right)\left(1_{H}\right) a_{i}$.

$\Leftarrow$ : Suppose $\gamma^{-1}$ is the inverse of $\gamma$, define $i^{\prime}:{ }^{*}() \rightarrow()^{*}$ by

$$
i_{M}^{\prime}(f)(m):=f\left(\gamma^{-1} \cdot m\right)
$$

for any $m \in M \in \operatorname{Rep}(H), f \in{ }^{*} M$. Obviously $i^{\prime}$ is the inverse of $i$.

Since the objects in $\operatorname{Rep}(H)$ are all finite dimensional, we obtain that $V^{*} \otimes W^{*}=(W \otimes V)^{*}$ for any $V, W \in \operatorname{Rep}(H)$, which means ()$_{2}^{*}=i d$. Similarly, ${ }^{*}()_{2}=i d$. Thus if $i \in \Omega$ is a monoidal natural transformation, we have $i_{V} \otimes i_{W}=i_{V \otimes W}$.

LEMMA 2.4. $i$ is a monoidal natural transformation if and only if $\gamma$ is invertible and satisfies $\Delta(\gamma)=(\gamma \otimes \gamma) \Delta\left(1_{H}\right)$.

Proof. It is a direct computation to check that $i$ is monoidal iff $\gamma$ satisfies $\Delta(\gamma)=(\gamma \otimes \gamma) \Delta\left(1_{H}\right)$. From Lemma 2.3, $i$ is an isomorphism if and only if $\gamma$ is invertible. Thus the conclusion holds.

Definition 2.5. Let $H$ be a finite dimensional weak Hopf algebra with the bijective antipode $S$. A sovereign element of $H$ is a group-like element $\gamma$ such that $S^{2}(h)=\gamma h \gamma^{-1}$ for any $h \in H$. A sovereign weak Hopf algebra is a weak Hopf algebra with a sovereign element.

THEOREM 2.6. Let $H$ be a finite dimensional weak Hopf algebra with the bijective antipode $S$. Then $H$ is a sovereign weak Hopf algebra if and only if $\operatorname{Rep}(H)$ is a sovereign category.

Proof. Let $\gamma \in H$. If $\Delta(\gamma)=(\gamma \otimes \gamma) \Delta\left(1_{H}\right), \gamma$ is invertible and $S^{2}(h)=$ $\gamma h \gamma^{-1}$ for any $h \in H$, we immediately get that $(\gamma \otimes \gamma) \Delta\left(1_{H}\right)=\Delta\left(1_{H}\right)(\gamma \otimes \gamma)$. Thus from Lemma 2.1-Lemma 2.4, we obtain the conclusion. 
Assume that $(H, R)$ is a finite dimensional quasitriangular weak Hopf algebra. Define the Drinfeld element $u=S\left(R^{(2)}\right) R^{(1)}$. Recall from [15] that the following identities hold

$$
u^{-1}=R^{(2)} S^{2}\left(R^{(1)}\right), \quad \Delta(u)=\bar{R} \bar{R}_{21}(u \otimes u)=(u \otimes u) \bar{R} \bar{R}_{21},
$$

and

$$
S(u)=R^{(1)} S\left(R^{(2)}\right), \quad S^{2}(h)=u h u^{-1}
$$

for all $h \in H$.

Recall from [[15], Proposition 7.3] that $\operatorname{Rep}(H)$ is a ribbon category if and only if $H$ is a ribbon weak Hopf algebra, and Deligne's Theorem said that the twist is equivalent to a sovereign structure in an autonomous category. Thus we get the following theorem.

THEOREM 2.7. Let $(H, R)$ be a finite dimensional quasitriangular weak Hopf algebra with the bijective antipode $S$. Let $h=u^{-1} S(u)$. Then $H$ has a ribbon element if and only if there exists a sovereign element $\gamma \in H$, such that $\gamma^{2}=h^{-1}$.

Proof. Let $\mathbf{E}=\left\{\gamma \in G(H) \mid \gamma^{2}=h^{-1}, S^{2}(x)=\gamma x \gamma^{-1}, \forall x \in H\right\}$, and $\mathbf{F}$ be the collection of the ribbon elements in $H$. Define a map $P^{\prime}: \mathbf{E} \rightarrow \mathbf{F}$ by $P^{\prime}(\gamma)=$ $u^{-1} \gamma$. On the one hand, since $S^{2}(x)=u x u^{-1}$ for any $x \in H$, we have $u^{-1} \gamma x=$ $x u^{-1} \gamma$ which implies $u^{-1} \gamma$ is the central element in $H$.

On the other hand, recall from [[15], Proposition 5.7] that $\left(H^{o p, c o p}, \bar{R}_{21}\right)$ is also a quasitriangular weak Hopf algebra with the Drinfeld element $u^{-1}$, thus we immediately get

$$
\Delta\left(u^{-1}\right)=\left(u^{-1} \otimes u^{-1}\right) R_{21} R=R_{21} R\left(u^{-1} \otimes u^{-1}\right) .
$$

Hence we have

$$
\begin{aligned}
\Delta\left(u^{-1} \gamma\right) & =\Delta\left(u^{-1}\right) \Delta(\gamma) \\
& =\Delta\left(u^{-1}\right) \Delta\left(1_{H}\right)(\gamma \otimes \gamma) \\
& =R_{21} R\left(u^{-1} \gamma \otimes u^{-1} \gamma\right),
\end{aligned}
$$

and

$$
\begin{aligned}
S\left(u^{-1} \gamma\right) & =\gamma^{-1} S(u)^{-1}=\gamma u^{-1} \\
& =u^{-1} S^{2}(\gamma)=u^{-1} \gamma
\end{aligned}
$$

for any $\gamma \in \mathbf{E}$. That means $u^{-1} \gamma \in \mathbf{F}$, i.e., $P^{\prime}$ is well defined.

Conversely, define $Q^{\prime}: \mathbf{F} \rightarrow \mathbf{E}$ by $Q^{\prime}(v)=u v$ for any $v \in \mathbf{F}$. Firstly, from [[15], Lemma A.1], we have $v^{2}=u^{-1} S(u)^{-1}$. Thus we get

$$
(u v)^{2}=u v^{2} u=S(u)^{-1} u=h^{-1} .
$$


Secondly, we have

$$
\begin{aligned}
\Delta(u v) & =\Delta(u) \Delta(v)=(u \otimes u) \bar{R} \Delta^{o p}(1) R(v \otimes v) \\
& =(u \otimes u) \Delta(1)(v \otimes v) \\
& =(u v \otimes u v) \Delta(1) \quad(\text { Since } v \text { is a central element }) \\
& =\Delta(1)(u v \otimes u v) \quad\left(\text { Since }(u \otimes u) \Delta\left(1_{H}\right)=\Delta\left(1_{H}\right)(u \otimes u)\right) .
\end{aligned}
$$

Since $u v$ is invertible, $u v \in G(H)$.

At last, by $v \in Z(H)$, it is easy to get that $S^{2}(x)=u v x(u v)^{-1}$. Thus $Q^{\prime}$ is well defined, and it is the inverse of $P^{\prime}$.

Dually, we have the following definition and theorems which generalize [[1], Definition 2.7] and [[1], Proposition $2.9 \& 2.10]$, respectively.

Definition 2.8. Suppose that $H$ is a finite dimensional weak Hopf algebra with the bijective antipode $S$. A sovereign character on $H$ is a dual group-like linear form $\beta$ such that $S^{2}=\beta * i d * \beta^{-1}$. A cosovereign weak Hopf algebra is a weak Hopf algebra with a sovereign character on it.

THEOREM 2.9. Let $H$ be a finite dimensional weak Hopf algebra with the bijective antipode $S$. Then $H$ is a cosovereign weak Hopf algebra if and only if $\operatorname{Corep}(H)$ is a sovereign category.

Thus we get the following theorem which is dual to Theorem 2.7.

THEOREM 2.10. Let $(H, \sigma)$ be a finite dimensional weak Hopf algebra with the bijective antipode $S$ and the coquasitriangular structure $\sigma$. Then $H$ is a coribbon weak Hopf algebra with the ribbon form $\tau$ if and only if there exists a sovereign character $\beta: H \rightarrow k$, such that $\beta * \beta=\left(\lambda^{-1} \circ S\right) * \lambda$.

\section{Applications}

In this section we give some examples of ribbon weak Hopf algebras and sovereign weak Hopf algebras. The examples of coribbon weak Hopf algebras and cosovereign weak Hopf algebras can be obtained by the duality.

Let $H$ be a finite dimensional weak Hopf algebra. On the $k$-linear space $H^{* \operatorname{cop}} \otimes_{k} H$ define a multiplication by

$$
(\phi \otimes h)(\varphi \otimes g)=\phi \varphi\left(S^{-1}\left(h_{3}\right) ? h_{1}\right) \otimes h_{2} g,
$$

where $\phi, \varphi \in H^{* c o p}, g, h \in H$. The linear span $I$ of the elements

$$
\begin{aligned}
& \phi \otimes \varepsilon_{s}(x) g-\phi(\varepsilon-x) \otimes g, \\
& \phi \otimes \varepsilon_{t}(x) g-\phi(x-\varepsilon) \otimes g,
\end{aligned}
$$


is a two-sided ideal in $H^{* c o p} \otimes_{k} H$. Let $D(H)$ be the factor-algebra $\left(H^{* c o p} \otimes_{k} H\right) / I$ and $\phi \otimes h$ denote the class of $\phi \otimes h$ in $D(H)$. Then recall from [2] that $D(H)$ is a weak Hopf algebra with unit $\varepsilon \otimes 1$, and its comultiplication, counit, and antipode are given by

$$
\begin{gathered}
\bar{\Delta}(\phi \bigotimes h)=\left(\phi_{2} \bowtie h_{1}\right)\left(\phi_{1} \bowtie h_{2}\right), \\
\bar{\varepsilon}(\phi \bigotimes h)=\phi\left(1_{1}\right) \varepsilon\left(1_{2} h\right), \\
\bar{S}(\phi \bigotimes h)=(\varepsilon \bigotimes S(h))\left(\hat{S}^{-1}(\phi) \bigotimes 1\right) .
\end{gathered}
$$

Recall from [15] that $D(H)$ is a quasitriangular weak Hopf algebra with the $R$-matrix $R=\sum_{i}\left(\varepsilon \otimes e_{i}\right) \otimes\left(e^{i} \otimes 1\right)$, where $e_{i}$ and $e^{i}$ are dual bases in $H$ and $H^{*}$. Thus the Drinfeld element in $D(H)$ is $u=\hat{S}^{-1}\left(e^{i}\right) \bigotimes e_{i}$.

\subsection{The Drinfeld double of a 2-dimensional weak Hopf algebra} structures:

Let $H_{2}$ be a weak Hopf algebra with a basis $\{1, e\}$ and the following

$\mathrm{H} 21)$ the multiplication is given by

\begin{tabular}{|c|l|l|}
\hline$H_{2}$ & 1 & $e$ \\
\hline 1 & 1 & $e$ \\
$e$ & $e$ & $e$ \\
\hline
\end{tabular}

$\mathrm{H} 22)$ the comultiplication is given by

$$
\Delta(1)=(1-e) \otimes(1-e)+e \otimes e, \quad \Delta(e)=e \otimes e ;
$$

$\mathrm{H} 23)$ the counit is given by

$$
\varepsilon(1)=2, \quad \varepsilon(e)=1 ;
$$

H24) the antipode is given by $S=i d$.

It is easy to get that $\varepsilon_{s}=\varepsilon_{t}=i d$.

Suppose that the dual basis of $\{1, e\}$ in $H_{2}^{*}$ is $\{I, E\}$, thus $H_{2}^{*}$ have a weak Hopf algebra structure as follows:

$\mathrm{CH} 21)$ the multiplication is given by

\begin{tabular}{|c|c|c|}
\hline$H_{2}^{*}$ & $I$ & $E$ \\
\hline$I$ & $I$ & $-I$ \\
$E$ & $-I$ & $2 I+E$ \\
\hline
\end{tabular}

$\mathrm{CH} 22)$ the comultiplication is given by

$$
\hat{\Delta}(I)=I \otimes I, \quad \hat{\Delta}(E)=I \otimes E+E \otimes I+E \otimes E ;
$$

$\mathrm{CH} 23$ ) the unit element is $1_{H_{2}^{*}}=\varepsilon=2 I+E$; 
$\mathrm{CH} 24)$ the counit is given by

$$
\hat{\varepsilon}(I)=1, \quad \hat{\varepsilon}(E)=0 ;
$$

$\mathrm{CH} 25)$ the antipode is given by $\hat{S}=i d$.

Proposition 3.1. $\mathrm{H}_{2}^{*}$ is isomorphic to $\mathrm{H}_{2}$ as weak Hopf algebras.

Proof. Define a map $\theta: H_{2} \rightarrow H_{2}^{*}$ by

$$
\theta(1)=2 I+E, \quad \theta(e)=I+E .
$$

It is easy to check that $\theta$ is an isomorphism of weak Hopf algebras.

Now consider $D\left(H_{2}\right)=H_{2}^{* c o p} \bowtie H_{2}$. Since $\phi \bigotimes \varepsilon_{s}(x) g=\phi(\varepsilon \leftarrow x) \bowtie g$ for any $\phi \in H_{2}^{*}, x, g \in H_{2}$, we can get

$$
\begin{gathered}
I \bowtie e=I \bowtie e \cdot 1=I(\varepsilon-e) \bowtie 1=0, \\
E \bowtie e=E \bowtie e \cdot 1=E(\varepsilon-e) \bowtie 1=I \bowtie 1+E \bowtie 1,
\end{gathered}
$$

which implies $\{I \otimes 1, E \otimes 1\}$ is a basis of $D\left(H_{2}\right)$.

Furthermore, it is easy to get that $D\left(H_{2}\right)$ is isomorphic to $H_{2}^{* c o p}=H_{2}$ by the canonical homomorphism $j_{H}: H_{2}^{* c o p} \rightarrow D\left(H_{2}\right)$ defined by $j_{H}(\phi)=\phi 区 1$ for all $\phi \in H_{2}^{* c o p}$.

THEOREM 3.2. $\mathrm{H}_{2}$ is a ribbon weak Hopf algebra.

Proof. Since $D\left(H_{2}\right)$ is isomorphic to $H_{2}$ via $I \bowtie 1 \mapsto e, E \bowtie 1 \mapsto 1-2 e$, and $D\left(H_{2}\right)$ is a quasitriangular weak Hopf algebra with the $R$-matrix

$$
R=(\varepsilon \bigotimes 1) \otimes(I \otimes 1)+(I \bowtie 1) \otimes(E \bowtie 1)+(E \bowtie 1) \otimes(E \bowtie 1),
$$

then $H_{2}$ is also a quasitriangular weak Hopf algebra with the $R$-matrix $R=\Delta(1)$. Furthermore, the Drinfeld element $u=1$.

Obviously 1 is the unique group-like element in $H_{2}$ and satisfies Theorem 2.7. Thus $H_{2}$ is a ribbon weak Hopf algebra with the ribbon element 1.

\subsection{The Drinfeld double of a 3-dimensional weak Hopf algebra}

Let $H_{3}$ be a weak Hopf algebra with a basis $\{1, e, f\}$ where

$\mathrm{H} 31)$ the multiplication is given by

\begin{tabular}{|c|c|c|c|}
\hline$H_{3}$ & 1 & $e$ & $f$ \\
\hline 1 & 1 & $e$ & $f$ \\
$e$ & $e$ & $e$ & $f$ \\
$f$ & $f$ & $f$ & $f$ \\
\hline
\end{tabular}


H32) the comultiplication is given by

$$
\begin{gathered}
\Delta(1)=(1-e) \otimes(1-e)+e \otimes e, \\
\Delta(e)=e \otimes e, \quad \Delta(f)=e \otimes f+f \otimes e-2 f \otimes f ;
\end{gathered}
$$

$\mathrm{H} 33)$ the counit is given by

$$
\varepsilon(1)=2, \quad \varepsilon(e)=1, \quad \varepsilon(f)=0 ;
$$

H34) the antipode is given by $S=i d$.

It is easy to get that in $H_{3}$

$$
\varepsilon_{S}(1)=\varepsilon_{t}(1)=1, \quad \varepsilon_{s}(e)=\varepsilon_{t}(e)=e, \quad \varepsilon_{s}(f)=\varepsilon_{t}(f)=0 .
$$

Suppose that the dual basis of $\{1, e, f\}$ in $H_{3}^{*}$ is $\{I, E, F\}$, thus $H_{3}^{*}$ has a weak Hopf algebra structure:

$\mathrm{CH} 31)$ the multiplication is given by

\begin{tabular}{|c|c|c|c|}
\hline$H_{3}^{*}$ & $I$ & $E$ & $F$ \\
\hline$I$ & $I$ & $-I$ & 0 \\
$E$ & $-I$ & $2 I+E$ & $F$ \\
$F$ & 0 & $F$ & $-2 F$ \\
\hline
\end{tabular}

$\mathrm{CH} 32)$ the comultiplication is given by

$$
\begin{gathered}
\hat{\Delta}(I)=I \otimes I, \quad \hat{\Delta}(E)=I \otimes E+E \otimes I+E \otimes E, \\
\hat{\Delta}(F)=I \otimes F+F \otimes I+E \otimes F+F \otimes E+F \otimes F ;
\end{gathered}
$$

$\mathrm{CH} 33)$ the unit element is $1_{H_{3}^{*}}=\varepsilon=2 I+E$;

$\mathrm{CH} 34)$ the counit is given by

CH35) the antipode $\hat{S}=i d$.

$$
\hat{\varepsilon}(I)=1, \quad \hat{\varepsilon}(E)=\hat{\varepsilon}(F)=0 ;
$$

Remark. $H_{3}^{*}$ and $H_{3}$ are isomorphic as weak Hopf algebras. The bijection $\beta: H_{3} \rightarrow H_{3}^{*}$ is given by

$$
\beta(1)=2 I+E, \quad \beta(e)=I+E, \quad \beta(f)=-\frac{1}{2} F .
$$

Now consider $D\left(H_{3}\right)=H_{3}^{* c o p} \bowtie H_{3}$. Since $\phi \bigotimes \varepsilon_{s}(x) g=\phi(\varepsilon \leftarrow x) \bowtie g$ for any $\phi \in H_{3}^{*}, x, g \in H_{3}$, we can get

$$
I \bowtie e=I \bowtie e \cdot 1=I(\varepsilon-e) \bowtie 1=0,
$$

$I \bowtie f=I \bowtie e \cdot f=I(\varepsilon-e) \bowtie f=0$,

$$
\begin{aligned}
& E \bowtie e=E \bowtie e \cdot 1=E(\varepsilon-e) \bowtie 1=I \bowtie 1+E \bowtie 1, \\
& F \bowtie e=F \bowtie e \cdot 1=F(\varepsilon-e) \bowtie 1=F \bowtie 1,
\end{aligned}
$$

which implies $\{I \otimes 1, E \otimes 1, E \bowtie f, F \bowtie 1, F \bowtie f\}$ is a basis of $D\left(H_{3}\right)$. 
Furthermore, the weak Hopf algebra structure of $D\left(H_{3}\right)$ is as follows: DH31) the multiplication and counit are given by

\begin{tabular}{|c|c|c|c|c|c|}
\hline$D\left(H_{3}\right)$ & $I \bowtie 1$ & $E \bowtie 1$ & $E \bowtie f$ & $F \bowtie 1$ & $F \bowtie f$ \\
\hline$I \bowtie 1$ & $I \bowtie 1$ & $-I \bowtie 1$ & 0 & 0 & 0 \\
\hline$E 区 1$ & $-I \otimes 1$ & $2 I \bowtie 1+E \bowtie 1$ & $E \bowtie f$ & $F \bowtie 1$ & $F \bowtie f$ \\
\hline$E \bowtie f$ & 0 & $E \bowtie f$ & $E \otimes f$ & $F \bowtie f$ & $F \bowtie f$ \\
\hline$F \otimes 1$ & 0 & $F \otimes 1$ & $F \otimes f$ & $-2 F \bowtie 1$ & $-2 F \bowtie f$ \\
\hline$F \bowtie f$ & 0 & $F \bowtie f$ & $F \bowtie f$ & $-2 F \bowtie f$ & $-2 F \bowtie f$ \\
\hline $\bar{\varepsilon}$ & 1 & 0 & 0 & 0 & 0 \\
\hline
\end{tabular}

DH32) the comultiplication is given by

$\bar{\Delta}(I \otimes 1)=(I \otimes 1) \otimes(I \bowtie 1)$,

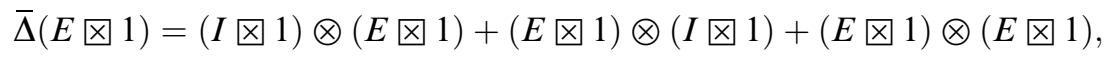

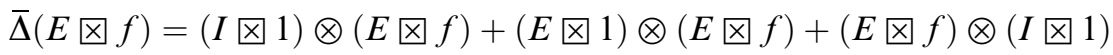

$+(E \otimes f) \otimes(E \otimes 1)-2(E \otimes f) \otimes(E \otimes f)$,

$\bar{\Delta}(F \bigotimes 1)=(I \otimes 1) \otimes(F \bowtie 1)+(E \bowtie 1) \otimes(F \bowtie 1)+(F \bigotimes 1) \otimes(I \bowtie 1)$

$+(F \bowtie 1) \otimes(E \otimes 1)+(F \bowtie 1) \otimes(F \bowtie 1)$,

$\bar{\Delta}(F \bigotimes f)=(I \rrbracket 1) \otimes(F \bowtie f)+(E \otimes 1) \otimes(F \bigotimes f)+(E \bowtie f) \otimes(F \bowtie 1)$

$-2(E \otimes f) \otimes(F \bowtie f)+(F \bigotimes 1) \otimes(E \otimes f)$

$+(F \bowtie f) \otimes(I \otimes 1)+(F \bowtie f) \otimes(E \bowtie 1)$

$-2(F \bowtie f) \otimes(E \bowtie f)+(F \bowtie 1) \otimes(F \bowtie f)$

$+(F 区 f) \otimes(F \bowtie 1)-2(F \bowtie f) \otimes(F 区 f) ;$

DH33) the antipode is given by $\bar{S}=i d$.

TheOREM 3.3. $D\left(\mathrm{H}_{3}\right)$ is a ribbon weak Hopf algebra.

Proof. It is straightforward to check that $G\left(D\left(H_{3}\right)\right)$ is the product of two cyclic groups of order 2. Actually, the group-like elements in $D\left(H_{3}\right)$ are

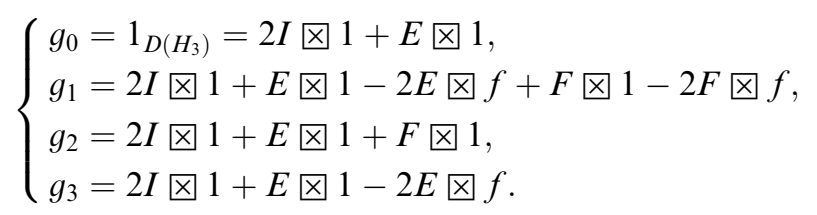


and they satisfy Theorem 2.7 since the Drinfeld element of $D\left(H_{3}\right)$ is

$$
u=2 I \bowtie 1+E \bowtie 1+F \bowtie f .
$$

Thus $D\left(H_{3}\right)$ is a ribbon weak Hopf algebra. Moreover, the ribbon elements in $D\left(H_{3}\right)$ are

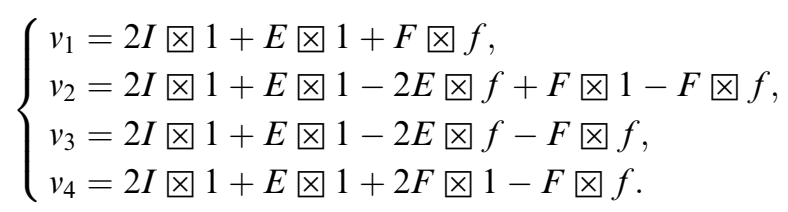

3.3. The Drinfeld double of the Sweedler's 5-dimensional weak Hopf algebra

Let $H_{5}$ be the Sweedler's 5-dimensional weak Hopf algebra with a basis $\{1, e, c, x, y\}$ satisfying the following:

$\mathrm{H} 51)$ the multiplication is given by

\begin{tabular}{|c|c|c|c|c|c|}
\hline$H_{5}$ & 1 & $e$ & $c$ & $x$ & $y$ \\
\hline 1 & 1 & $e$ & $c$ & $x$ & $y$ \\
$e$ & $e$ & $e$ & $c$ & $x$ & $y$ \\
$c$ & $c$ & $c$ & $e$ & $-y$ & $-x$ \\
$x$ & $x$ & $x$ & $y$ & 0 & 0 \\
$y$ & $y$ & $y$ & $x$ & 0 & 0 \\
\hline
\end{tabular}

H52) the comultiplication is given by

$$
\begin{aligned}
& \Delta(1)=1 \otimes 1-1 \otimes e-e \otimes 1+2 e \otimes e, \\
& \Delta(e)=e \otimes e, \quad \Delta(c)=c \otimes c, \\
& \Delta(x)=c \otimes x+x \otimes e, \quad \Delta(y)=e \otimes y+y \otimes c ;
\end{aligned}
$$

H53) the counit is given by

$$
\varepsilon(1)=2, \quad \varepsilon(e)=\varepsilon(c)=1, \quad \varepsilon(x)=\varepsilon(y)=0 ;
$$

H54) the antipode is given by

$$
S(1)=1, \quad S(e)=e, \quad S(c)=c, \quad S(x)=y, \quad S(y)=-x .
$$

It is easy to get that

$$
\begin{gathered}
\varepsilon_{s}(1)=\varepsilon_{t}(1)=1, \quad \varepsilon_{s}(e)=\varepsilon_{t}(e)=e, \\
\varepsilon_{s}(c)=\varepsilon_{t}(c)=e, \quad \varepsilon_{s}(x)=\varepsilon_{t}(x)=\varepsilon_{s}(y)=\varepsilon_{t}(y)=0 .
\end{gathered}
$$

Suppose that the dual basis of $\{1, e, c, x, y\}$ in $H_{5}^{*}$ is $\{I, E, C, X, Y\}$, then $H_{5}^{*}$ has a weak Hopf algebra structure as follows: 
CH51) the multiplication is given by

\begin{tabular}{|c|c|c|c|c|c|}
\hline$H_{5}^{*}$ & $I$ & $E$ & $C$ & $X$ & $Y$ \\
\hline$I$ & $I$ & $-I$ & 0 & 0 & 0 \\
$E$ & $-I$ & $2 I+E$ & 0 & 0 & $Y$ \\
$C$ & 0 & 0 & $C$ & $X$ & 0 \\
$X$ & 0 & $X$ & 0 & 0 & 0 \\
$Y$ & 0 & 0 & $Y$ & 0 & 0 \\
\hline
\end{tabular}

CH52) the comultiplication is given by

$$
\begin{aligned}
& \hat{\Delta}(I)=I \otimes I, \\
& \hat{\Delta}(E)=E \otimes E+I \otimes E+E \otimes I+C \otimes C, \\
& \hat{\Delta}(C)=I \otimes C+C \otimes I+E \otimes C+C \otimes E, \\
& \hat{\Delta}(X)=I \otimes X+X \otimes I+E \otimes X+X \otimes E+Y \otimes C-C \otimes Y, \\
& \hat{\Delta}(Y)=I \otimes Y+Y \otimes I+E \otimes Y+Y \otimes E+X \otimes C-C \otimes X ;
\end{aligned}
$$

CH53) the unit element is $1_{H_{5}^{*}}=\varepsilon=2 I+E+C$;

$\mathrm{CH} 54)$ the counit is given by

$$
\hat{\varepsilon}(I)=1, \quad \hat{\varepsilon}(E)=\hat{\varepsilon}(C)=\hat{\varepsilon}(X)=\hat{\varepsilon}(Y)=0 ;
$$

$\mathrm{CH} 55)$ the antipode is given by

$$
\hat{S}(I)=I, \quad \hat{S}(E)=E, \quad \hat{S}(C)=C, \quad \hat{S}(X)=-Y, \quad \hat{S}(Y)=X .
$$

Now consider $D\left(H_{5}\right)=H_{5}^{* \text { cop }} \bigotimes H_{5}$. Similar to $D\left(H_{2}\right)$ and $D\left(H_{3}\right)$, we can get that $D\left(H_{5}\right)$ is a 17-dimensional weak Hopf algebra with a basis $\left\{x_{00}=I \otimes 1\right.$, $x_{11}=E \otimes 1, x_{12}=E \bowtie c, x_{13}=E \otimes x, x_{14}=E \otimes y, x_{21}=C \bowtie 1, x_{22}=C \bowtie c$, $x_{23}=C \bowtie x, x_{24}=C \bowtie y, x_{31}=X \bowtie 1, x_{32}=X \bowtie c, x_{33}=X \bowtie x, x_{34}=X \bowtie y$, $\left.x_{41}=Y \otimes 1, x_{42}=Y \bowtie c, x_{43}=Y \bowtie x, x_{44}=Y \bowtie y\right\}$.

Furthermore, the weak Hopf algebra structure of $D\left(H_{5}\right)$ is given as follows:

DH51) since $\phi \bigotimes h=\left(\phi \bigotimes 1_{H}\right)(\varepsilon \otimes h)$ for all $\phi \in H^{* c o p}$ and $h \in H$, and there are two canonical weak Hopf algebra homomorphisms: $i_{H}: H \rightarrow D(H)$ defined by $i_{H}(h)=\varepsilon \otimes h$ and $j_{H}: H^{* c o p} \rightarrow D(H)$ defined by $j_{H}(\phi)=\phi \bigotimes 1_{H}$, we only need to give the products under the form $(\varepsilon \otimes h)\left(\phi \bigotimes 1_{H}\right)$. Moreover, we have

$$
\begin{aligned}
& (\psi \bigotimes a)(\varphi \rrbracket b)=\left(\psi \bowtie 1_{H}\right)(\varepsilon \bigotimes a)\left(\varphi \rrbracket 1_{H}\right)(\varepsilon \rrbracket b) \\
& =\left(\psi \bowtie 1_{H}\right)\left(f \otimes 1_{H}\right)(\varepsilon \otimes c)(\varepsilon \bowtie b) \\
& =\left(\psi f \otimes 1_{H}\right)(\varepsilon \bowtie c b),
\end{aligned}
$$


for any $\psi, \varphi \in H^{* c o p}, a, b \in H$, where $f \bowtie c=(\varepsilon \otimes a)\left(\varphi \otimes 1_{H}\right)$ obtained by following

\begin{tabular}{|c|c|c|c|c|c|}
\hline$D\left(H_{5}\right)$ & $I \bowtie 1$ & $E \bowtie 1$ & $C \bowtie 1$ & $X \bowtie 1$ & $Y \bowtie 1$ \\
\hline$\varepsilon \bigotimes 1$ & $I \bowtie 1$ & $E \bowtie 1$ & $C \bowtie 1$ & $X \bowtie 1$ & $Y \otimes 1$ \\
\hline$\varepsilon \bowtie e$ & 0 & $E \otimes c$ & $C \bowtie c$ & $-X \bowtie c$ & $-Y \bowtie c$ \\
\hline$\varepsilon \bowtie c$ & 0 & $E \otimes c$ & $C \bowtie c$ & $-X \square c$ & $-Y \overline{\mathrm{\Xi}} c$ \\
\hline$\varepsilon \bigotimes x$ & 0 & $C \bowtie x$ & $E \bowtie x$ & $\begin{array}{c}I \bowtie 1+E \bowtie 1 \\
-E \bowtie c+y \rrbracket x\end{array}$ & $\begin{array}{c}-C \bowtie c-C \bowtie 1 \\
+X \bowtie x\end{array}$ \\
\hline$\varepsilon \bowtie y$ & 0 & $C \bowtie y$ & $E \bowtie y$ & $\begin{array}{c}I \bowtie 1+E \bowtie 1 \\
-E \bowtie c-Y \bowtie y\end{array}$ & $\begin{array}{c}C \bowtie c+C \bowtie 1 \\
-X \rrbracket y\end{array}$ \\
\hline
\end{tabular}

DH52) since $\bar{\Delta}(\phi \bigotimes h)=\bar{\Delta}(\phi 区 1) \bar{\Delta}(\varepsilon \rrbracket h)$, we only need to give the coproducts under the form $\bar{\Delta}(\phi 区 1)$ and $\bar{\Delta}(\varepsilon \otimes h)$ :

$\bar{\Delta}(I \otimes 1)=(I \otimes 1) \otimes(I \bowtie 1)$,

$\bar{\Delta}(E \otimes 1)=(I \otimes 1) \otimes(E \bowtie 1)+(E \bowtie 1) \otimes(I \otimes 1)$

$+(E \otimes 1) \otimes(E \otimes 1)+(C \otimes 1) \otimes(C \otimes 1)$,

$\bar{\Delta}(C \otimes 1)=(I \otimes 1) \otimes(C \bigotimes 1)+(E \otimes 1) \otimes(C \bowtie 1)$

$+(C \otimes 1) \otimes(I \otimes 1)+(C \bowtie 1) \otimes(E \bowtie 1)$,

$\bar{\Delta}(X \bigotimes 1)=(X \bigotimes 1) \otimes(I \bowtie 1)+(X \bowtie 1) \otimes(E \bowtie 1)+(C \bowtie 1) \otimes(Y \bigotimes 1)$

$-(Y \otimes 1) \otimes(C \bowtie 1)+(E \otimes 1) \otimes(X \bowtie 1)+(I \otimes 1) \otimes(X \bowtie 1)$,

$\bar{\Delta}(Y \otimes 1)=(I \otimes 1) \otimes(Y \bigotimes 1)+(E \otimes 1) \otimes(Y \bigotimes 1)+(Y \bigotimes 1) \otimes(I \otimes 1)$

$+(Y \otimes 1) \otimes(E \otimes 1)+(C \otimes 1) \otimes(X \bigotimes 1)-(X \otimes 1) \otimes(C \otimes 1)$,

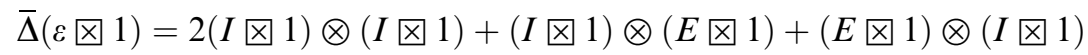

$+(E \bowtie 1) \otimes(E \bowtie 1)+(C \bowtie 1) \otimes(C \bowtie 1)$

$+(I \otimes 1) \otimes(C \bowtie 1)+(E \otimes 1) \otimes(C \bowtie 1)$

$+(C \bowtie 1) \otimes(I \otimes 1)+(C \bowtie 1) \otimes(E \bowtie 1)$,

$\bar{\Delta}(\varepsilon \bigotimes e)=(I \otimes 1) \otimes(I \bowtie 1)+(I \bowtie 1) \otimes(E \bowtie 1)+(E \bowtie 1) \otimes(I \rrbracket 1)$

$+(E \bowtie 1) \otimes(E \bowtie 1)+(C \bowtie 1) \otimes(C \bowtie 1)$

$+(I \otimes 1) \otimes(C \bowtie 1)+(E \otimes 1) \otimes(C \bowtie 1)$

$+(C \otimes 1) \otimes(I \otimes 1)+(C \bowtie 1) \otimes(E \bowtie 1)$,

$\bar{\Delta}(\varepsilon \otimes c)=(E \otimes c) \otimes(E \otimes c)+(C \bigotimes c) \otimes(C \bigotimes c)$

$+(C \otimes c) \otimes(E \otimes c)+(E \otimes c) \otimes(C \otimes c)$, 


$$
\begin{aligned}
& \bar{\Delta}(\varepsilon \rrbracket x)=(E \bowtie c) \otimes(E \bowtie x)+(E \bowtie x) \otimes(E \bowtie 1)+(E \bowtie x) \otimes(I \rrbracket 1) \\
& +(C \bigotimes c) \otimes(C \bowtie x)+(C \bowtie x) \otimes(C \bowtie 1)+(E \otimes c) \otimes(C \bigotimes x) \\
& +(C \bigotimes c) \otimes(E \otimes x)+(C \bowtie x) \otimes(E \otimes 1) \\
& +(C \bowtie x) \otimes(I \otimes 1)+(E \otimes x) \otimes(C \bowtie 1),
\end{aligned}
$$

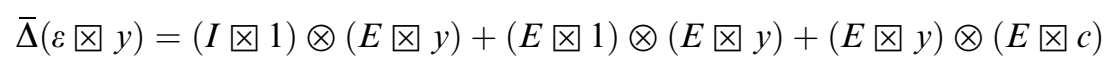

$$
\begin{aligned}
& +(C \otimes 1) \otimes(C \bowtie y)+(C \bowtie y) \otimes(C \bowtie c)+(E \otimes 1) \otimes(C \otimes y) \\
& +(I \otimes 1) \otimes(C \bowtie y)+(C \bowtie 1) \otimes(E \otimes y) \\
& +(C \otimes y) \otimes(E \bowtie c)+(E \bowtie y) \otimes(C \bowtie c) ;
\end{aligned}
$$

DH53) the unit element is $1_{D\left(H_{5}\right)}=2 I \bowtie 1+E \bowtie 1+C \bowtie 1$;

DH54) the counit is given by

$$
\begin{aligned}
& \bar{\varepsilon}(I \bowtie 1)=1, \quad \bar{\varepsilon}(E \bowtie c)=1, \\
& \bar{\varepsilon}\left(x_{i j}\right)=0, \quad \text { for any }(i, j) \neq(0,0) \text { and }(i, j) \neq(1,2) ;
\end{aligned}
$$

DH55) since $\bar{S}(\phi \bigotimes h)=(\varepsilon \otimes S(h))\left(\hat{S}^{-1}(\phi) \bigotimes 1\right)$, the antipode of $D\left(H_{5}\right)$ is immediately determined by H54), CH55) and the table of the product.

THEOREM 3.4. $D\left(H_{5}\right)$ is a sovereign weak Hopf algebra, but not a ribbon weak Hopf algebra.

Proof. On the one hand, it is a direct computation to check that $G\left(D\left(H_{5}\right)\right)$ is the product of two cyclic groups of order 2. Actually, the group-like elements in $D\left(H_{5}\right)$ are

$$
\begin{aligned}
& 1_{D\left(H_{5}\right)}=2 I \rrbracket 1+E \rrbracket 1+C \bowtie 1, \\
& g_{1}=I \bowtie 1+E \bowtie c+C \bowtie c, \\
& g_{2}=I \bowtie 1+E \bowtie c-C \bowtie c, \\
& g_{3}=2 I \bowtie 1+E \bowtie 1-C \bowtie 1,
\end{aligned}
$$

and $g_{1}, g_{3}$ are sovereign elements.

On the other hand, since the quasitriangular structure of $D\left(H_{5}\right)$ is $R=$ $\sum_{i}\left(\varepsilon \bigotimes e_{i}\right) \otimes\left(e^{i} \bigotimes 1\right)$, we can get that the Drinfeld element

$$
u=2 I \bowtie 1+E \bowtie 1+C \bowtie c-X \bowtie y+Y \bowtie x,
$$

and

$$
u^{-1}=2 I \bowtie 1+E \bowtie 1+C \bowtie c-X \bowtie x-Y \bowtie y
$$


Then we have

$$
u^{-1} \bar{S}(u)=I \bowtie 1+E \bowtie c-C \bowtie c+2 Y \bowtie y .
$$

Thus there is no group-like element $g$ in $D\left(H_{5}\right)$ satisfing $g^{2}=u^{-1} \bar{S}(u)$.

Acknowledgement. The authors would like to thank the referee for his/her valuable comments on this paper. This work is partially supported by the NSF of China (No. 11371088) and the NSF of JiangSu Province (No. BK2012736) and the Fundamental Research Funds for the Central Universities (No. 3207013906).

\section{REFERENCES}

[1] J. Bichon, Cosovereign Hopf algebras, J. Pure Appl. Algebra 157 (2001), 121-133.

[2] G. Böнм, Doi-Hopf modules over weak Hopf algebras, Comm. Algebra 28 (2000), 4687-4698.

[ 3 ] G. Böhm, F. Nill and K. SzlachánYI, Weak Hopf algebras-I. Integral theory and $C^{*}$-structure, J. Algebra 221 (1999), 385-438.

[4] A. Bruguières ANd A. Virelizier, Hopf monads, Adv. Math. 215 (2007), 679-722.

[5] J. Chen, Y. Zhang And S. WAng, Twisting theory for weak Hopf algebras, Appl. Math. J. Chinese Univ. 23 (2008), 91-100.

[6] B. Drabant, Notes on balanced categories and Hopf algebras, Quantum groups and Lie theory, London Math. Soc. lecture note series 290, Cambridge Univ. Press, Cambridge, 2001, 63-88.

[7] P. Etingof, S. Gelaki, D. Nikshych and V. Ostrik, Tensor categories, Lecture note for the MIT course, available at: www-math.mit.edu/etingof/tenscat.pdf.

[8] P. Freyd and D. N. Yetter, Coherence theorems via knot theory, J. Pure Appl. Algebra 78 (1992), 49-76.

[9] T. Hayashi, Face algebras I. A generalization of quantum group theory, J. Math. Soc. Japan 50 (1998), 293-315.

[10] C. KASSEL, Quantum groups, Springer-Verlag, New York, 1995.

[11] L. H. KAUfFMAN AND D. E. RADFORD, A necessary and sufficient condition for a finitedimensional Drinfel'd double to be a ribbon Hopf algebra, J. Algebra 159 (1993), 98-114.

[12] G. LiU, Q. Chen AND H. ZHU, Transmutation theory of a coquasitriangular weak Hopf algebra, Front. Math. China 6 (2011), 855-869.

[13] G. Maltsiniotis, Traces dans les catgories monoïdales, dualit et catgories monoïdales fibres, Cahiers de topologie et gomtrie differentielle catgoriques, 1995, 195-288.

[14] D. Nikshych, On the structure of weak Hopf algebras, Adv. Math. 170 (2002), 257-286.

[15] D. Nikshych, V. G. Turaev and L. Vainerman, Invariants of knots and 3-manifolds from quantum groupoids, Topology and its Applications 127 (2003), 91-123.

[16] D. Nikshych and L. Vainerman, Finite quantum groupoids and their applications, New directions in Hopf algebras, MSRI Publications 43 (2002), 211-262.

[17] H. Pfeiffer, Tannaka-Kreǐn reconstruction and a characterization of modular tensor categories, J. Algebra 321 (2009), 3714-3763.

[18] N. Reshetikhin and V. G. Turaev, Ribbon graphs and their invariants derived from quantum groups, Commun. Math. Phys. 127 (1990), 1-26.

[19] P. Selinger, A survey of graphical languages for monoidal categories, ArXiv: math.QA/ 0908.3347. 
[20] D. N. Yetter, Framed tangles and a theorem of Deligne on braided deformations of tannakian categories, Deformation theory and quantum groups with applications to mathematical physics, Contemporary mathematics 134, American Mathematical Society, 1992, $325-345$.

Xiaohui Zhang

Department of Mathematics

SOUTHEAST UNIVERSITY

JiANGSU NANJING 210096

P. R. CHINA

E-mail: zxhhhhh@gmail.com

Xiaofan Zhao

Department of Mathematics

SOUTHEAST UNIVERSITY

JiANGSU NANJING 210096

P. R. CHINA

E-mail: zhaoxiaofan8605@gmail.com

Shuanhong Wang

Department of Mathematics

SOUTHEAST UNIVERSITY

JiAngsu NANJing 210096

P. R. CHINA

E-mail: shuanhwang@yahoo.com 